\title{
Spectral and timing studies of 2S 1417-624 during a giant outburst
}

\author{
Shivangi Gupta ${ }^{1 \star}$, Sachindra Naik ${ }^{\dagger} \uparrow$, Gaurava K. Jaisawal ${ }^{2} \ddagger$, Prahlad R. Epili ${ }^{1} \oint$ \\ 1 Astronomy and Astrophysics Division, Physical Research Laboratory, Navrangapura, Ahmedabad - 380009, Gujarat, India \\ 2 National Space Institute, Technical University of Denmark, Elektrovej 327-328, DK-2800 Lyngby, Denmark
}

\begin{abstract}
We present the results obtained from timing and spectral studies of the accretion powered X-ray pulsar 2S 1417-624 during a giant outburst in 2009 by using Rossi Xray Timing Explorer (RXTE) observations. X-ray pulsations were detected in the light curves obtained from all epochs of observations. The pulsar was found to be spinningup during the outburst. The pulse profiles were observed to be strongly dependent on photon energy and luminosity. A double peaked profile at lower luminosity evolved into a triple peaked profile at the peak of the outburst which is further reverted back to a double peaked structure during the decay of the outburst. An anti-correlation was also observed between the pulse fraction and the source flux. The 3-70 keV energy spectrum of pulsar was well described with a power law modified with high energy cutoff model along with an iron fluorescence line at $6.4 \mathrm{keV}$. Based on the evolution of pulse profile, pulse fraction and spectral parameters across observed luminosity, we interpret our results in terms of changes in the pulsar beam configuration from sub-critical to super-critical regimes.
\end{abstract}

Key words: stars: neutron - pulsars: individual: 2S 1417-624 - X-rays: stars.

\section{INTRODUCTION}

Accretion powered X-ray pulsars were discovered in early seventies with Uhuru satellite soon after the long awaited discovery of neutron star in radio band (Hewish et al. 1968; Giacconi et al. 1971). These sources are accreting neutron stars that appeared to be one of the brightest X-ray objects in the sky due to their transient activity. Most of these sources belong to the class of high mass X-ray binaries (HMXBs), where a magnetized neutron star with magnetic field in the order of $\mathrm{B} \sim 10^{12} \mathrm{G}$ accretes matter from a supergiant or non-giant optical companion. Among HMXBs, Be/X-ray binaries represent about two-thirds of their population. The optical companion in these systems is a non-supergiant OB star that shows emission lines in opti$\mathrm{cal} /$ infrared spectra at some point of the evolution (Reig 2011). The presence of an equatorial circumstellar disk around the Be star is known to be the cause of the emission lines in the optical/infrared spectrum and observed infrared excess compared to the classical B stars (Porter \& Rivinius 2003; Reig 2011). The orbiting neutron star in the Be/X-ray

\footnotetext{
* E-mail: shivangi@prl.res.in

$\dagger$ snaik@prl.res.in

† gaurava@space.dtu.dk

$\S$ prahlad@prl.res.in
}

binary system captures significant amount of matter from the circumstellar disk at the periastron passage leading to Xray outbursts (Type I) lasting for a few days to a few weeks. The peak luminosity during these events reaches up to one or two order of magnitude higher $\left(\sim 10^{35-37} \mathrm{erg} \mathrm{s}^{-1}\right)$ than the quiescent luminosity (Stella et al. 1986). Another kind of $\mathrm{X}$-ray enhancement i.e. Type II outbursts are also observed from neutron stars in Be/X-ray binaries. These outbursts are rare and independent of orbital phases of the binary system. These outbursts usually cover a significant fraction of the orbit(s) and last for several weeks to months with a peak luminosity of $\sim 10^{38} \mathrm{erg} \mathrm{s}^{-1}$ (Negueruela et al. 1998; Paul \& Naik 2011).

The transient Be/X-ray binary pulsar 2S 1417-624 was discovered in 1978 with $S A S$-3 (Apparao et al. 1980). During early observations, X-ray pulsations at $\sim 17.5 \mathrm{~s}$ were detected in the source light curves (Kelley et al. 1981). A B-type star located at a distance of 1.4-11.1 kpc and within the search box provided by Einstein observatory was identified as the optical companion of the pulsar (Grindlay et al. 1984). Using BATSE monitoring data of six consecutive outbursts between 1994 August and 1995 July, the binary orbital parameters of the system such as orbital period $P_{\text {orb }}=42.12 \mathrm{~d}$, eccentricity $e=0.446$, semi major axis $a_{x}$ sin $i=188 \mathrm{lt}-\mathrm{s}, \omega=300^{\circ} .3$ and time of periastron passage $T=$ JD 2449714.12 were derived by Finger et al. (1996) (see also Raichur \& Paul 2010). 
These authors also reported a spin-up of the pulsar at a rate of $(3-6) \times 10^{-11} \mathrm{~Hz} \mathrm{~s}^{-1}$ and found a correlation between spin-up rate and pulsed flux.

After almost four years of quiescence, 2S 1417-624 went into a strong X-ray outburst in 1999 November which was subsequently followed by a series of four mini-outbursts between 1999 December and 2000 August. Using RXTE observations of the pulsar during these outbursts, intensity dependent pulse profiles and pulsed fraction were reported (İnam et al. 2004). The pulsar continuum spectrum in 3-20 $\mathrm{keV}$ range was described with a power law model modified with cutoff at higher energies. Observed variations in spectral and timing parameters were explained in terms of disc accretion except at low flux durations where a temporary accretion geometry change was speculated (İnam et al. 2004). A complex iron line in 6.4-6.8 keV range was also detected during 1999-2000 RXTE observations of the pulsar. Detection of complex iron line indicated that the emission was originated from cold iron atoms along with $\mathrm{H}$ or He-like ionized gas around the neutron star (İnam et al. 2004). A Chandra observation of the pulsar in quiescent phase showed a simple continuum spectrum that was described by a power law or a blackbody component at a temperature of $1.5 \mathrm{keV}$ (Tsygankov et al. 2017). Unusual high blackbody temperature suggests that the neutron star was accreting from a stable cold disk in the faint state. Recently, the pulsar was detected in a giant outburst in 2018 March at a flux of $\sim 350 \mathrm{mCrab}$ in $15-50 \mathrm{keV}$ range (Nakajima et al. 2018; Krimm et al. 2018). Prior to the 2018 outburst, a giant outburst was also detected in 2009 November with a peak intensity of $\sim 300$ mCrab with Swift/BAT (Fig. 1; Krimm et al. 2009). The pulsar was monitored at multiple epochs with the $R X T E$ during this giant outburst. Using these observations, we investigate the evolution of pulse profiles, accretion geometry and spectral shape of the pulsar during the 2009 outburst. The present paper describes the observation details and analysis methods in Section 2. A description on timing and spectral studies are presented in Section 3, followed by result and discussion in next section.

\section{OBSERVATIONS AND ANALYSIS}

The Rossi X-ray Timing Explorer (RXTE) was launched on 1995 December 30 in a low earth orbit. It monitored the Xray sky extensively for about 16 years before being decommissioned in 2012 January. The RXTE consisted of three sets of instruments: All Sky Monitor (ASM; Levine et al. 1996), Proportional Counter Array ( $P C A$; Jahoda et al. 1996) and High Energy X-ray Timing Experiment (HEXTE; Rothschild et al. 1998), that provided a broad-band coverage in $3-250 \mathrm{keV}$ range. The $A S M$ was sensitive in $1.5-12 \mathrm{keV}$ range. The $P C A$ unit consisted of five identical Xenon filled proportional counters operated in 2-60 keV energy range. The total effective area of PCA was $\sim 6500 \mathrm{~cm}^{2}$ at $6 \mathrm{keV}$. The PCA has an energy resolution of $<18 \%$ at $6 \mathrm{keV}$ along with a timing resolution of $1 \mu \mathrm{s}$. The third instrument, HEXTE was operated in the hard X-ray range of 15 to $250 \mathrm{keV}$. The HEXTE comprised of two set of clusters called A and B, rocking orthogonal to each other for the simultaneous measurement of source and background. Each cluster was made

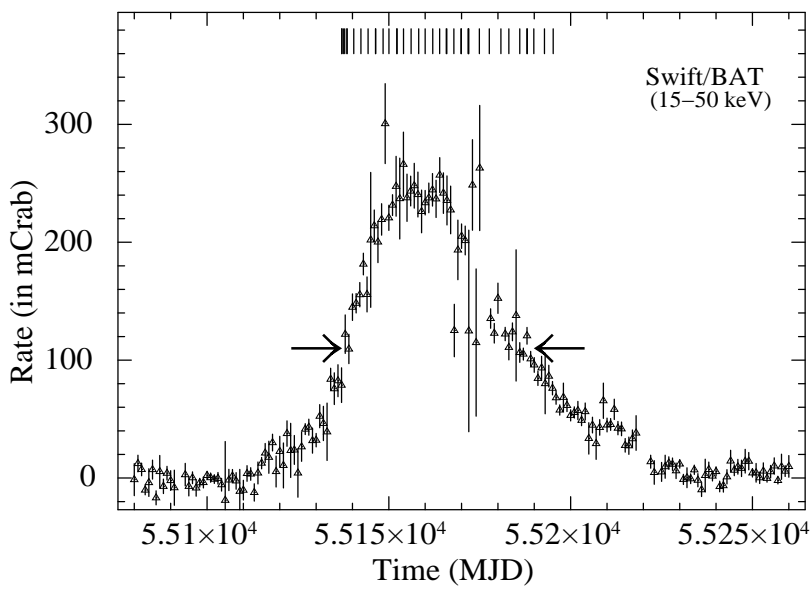

Figure 1. Swift/BAT monitoring light curve of 2S 1417-624 in $15-50 \mathrm{keV}$ range during a giant outburst in 2009. Arrow marks indicate the beginning and end of the RXTE pointed observations. A total of $39 R X T E$ pointed observations were made during this period and indicated by the vertical lines at the top of the figure.

up of four $\mathrm{NaI}(\mathrm{Tl}) / \mathrm{CsI}(\mathrm{Na})$ phoswich scintillation counters with a total collecting area of $\sim 1600 \mathrm{~cm}^{2}$.

We used a total of 39 pointed RXTE observations of the pulsar 2S 1417-624 carried out between 2009 November 2 to 2009 December 30. This covers an effective exposure of $\sim 133 \mathrm{ks}$ during the 2009 giant X-ray outburst. The dates of these observations are marked by vertical lines across the 15-50 keV light curve of the pulsar (Fig. 1) obtained from the Swift/BAT monitoring data (Krimm et al. 2013). Two additional horizontal arrows indicate the beginning and end of $R X T E$ pointed observations of the pulsar. The log of the observations is presented in Table 1.

We used Standard-1 and Standard-2 binned mode data from PCA detectors to investigate the pulse profile and spectral evolution of the pulsar. For this, HEASoft package of ver 6.16 was utilized along with the calibration data base of $R X T E$. We first created a good time interval file by applying filter selections on all available PCUs on individual observation. The filter selection was done by considering the electron contamination of $<0.2$, offset angle of $<0.02$ and elevation angle of $>10$ and other standard parameters. Using saextrct task, source light curve was extracted in $2-60 \mathrm{keV}$ range at a time resolution of $0.125 \mathrm{~s}$ from Standard- 1 data. Corresponding background light curve was also generated from Standard-2 data by using background model provided by the instrument team. Barycentric correction was applied on the background subtracted light curves to incorporate the motion of satellite and Earth to the barycenter of the solar system by using faxbary task of FTOOLS. For energy resolved light curves, we considered GoodXenon data by applying make_se task. The Standard-2 (PCA) and Cluster-B (HEXTE) mode data were used for carrying out spectral analysis. Phase-resolved spectroscopy was also performed by using GoodXenon data that have 256 energy channels. The response matrices were created by following the standard procedures. 

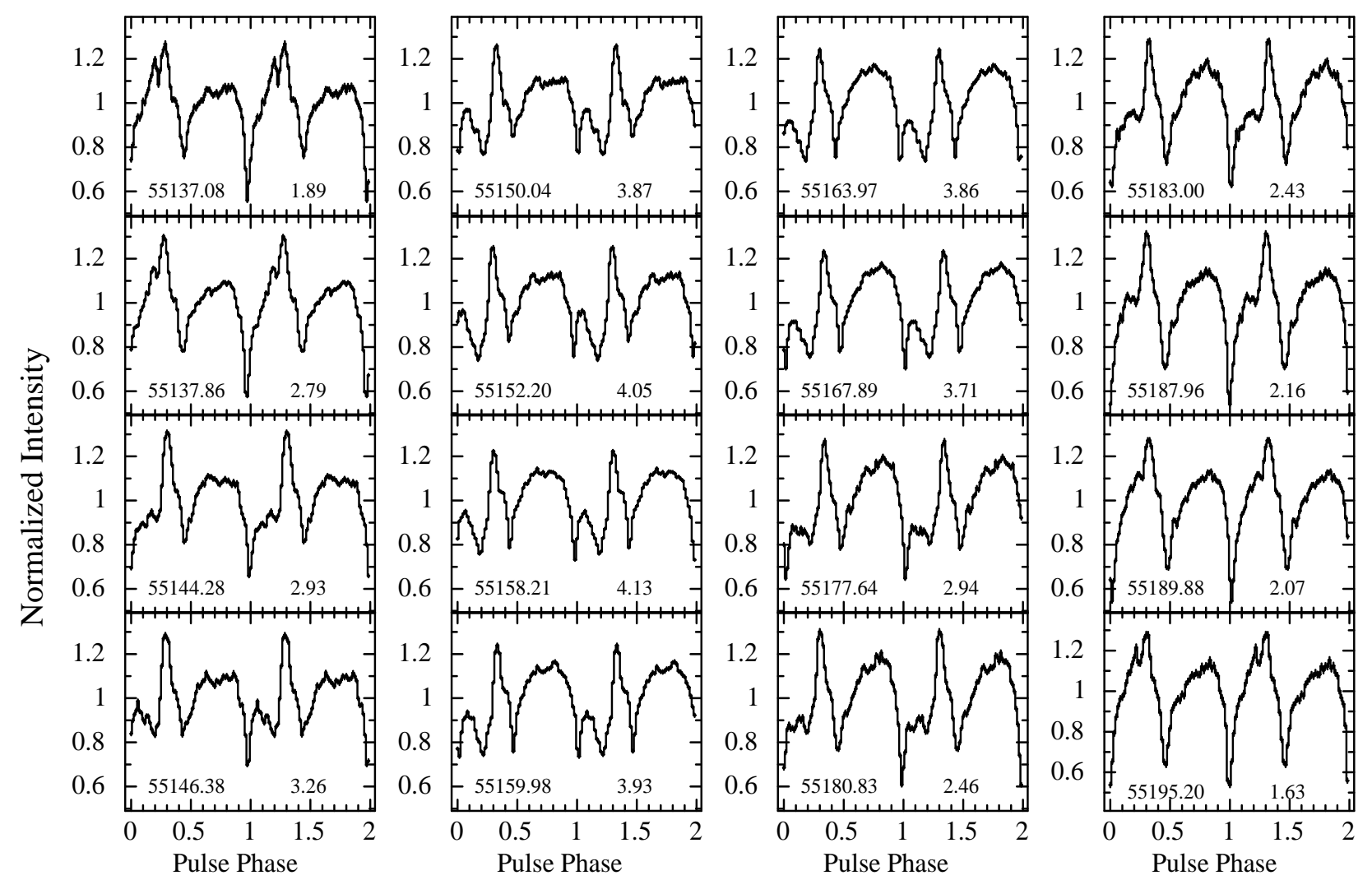

Figure 2. Pulse profiles of the pulsar at different phases of the 2009 giant X-ray outburst (from rise to decline). The profiles were generated by folding the $2-60 \mathrm{keV}$ light curves from PCA data at the respective spin periods. The numbers quoted on the left and right side of each panel indicate the beginning of the corresponding $R X T E$ pointed observation (in MJD) and the flux (in $10^{-9}$ erg $\mathrm{s}^{-1} \mathrm{~cm}^{-2}$ units), respectively. Two pulses are shown in each panel for clarity. The error-bars represent $1 \sigma$ uncertainties.

Table 1. Log of RXTE observations of the pulsar $2 \mathrm{~S}$ 1417-624 during 2009 outburst.

\begin{tabular}{cccc}
\hline $\begin{array}{c}\text { Proposal } \\
\text { ID }\end{array}$ & $\begin{array}{c}\text { Number of } \\
\text { Obs. (IDs) }\end{array}$ & $\begin{array}{c}\text { Time range } \\
(\mathrm{MJD})\end{array}$ & $\begin{array}{c}\text { Exposure Time } \\
(\mathrm{ksec})\end{array}$ \\
\hline & & & \\
94032 & 29 & $55137.08-55171.96$ & 98.52 \\
94444 & 10 & $55177.64-55195.20$ & 34.32 \\
\hline \multirow{2}{*}{ Total } & 39 & $55137-55195$ & 132.84 \\
\end{tabular}

\section{RESULTS}

\subsection{Timing analysis}

Source and background light curves were extracted from all the $R X T E / P C A$ pointed observations at a time resolution of $0.125 \mathrm{~s}$ by following procedures as described in Section 2 . Barycentric correction was also applied on the background subtracted light curves. Thereafter, the pulsation period of the pulsar was estimated by using a $\chi^{2}$-maximization technique (efsearch task of FTOOLS; Leahy 1987). Following this procedure, the barycentric corrected spin frequency of the pulsar was obtained from the light curves of all pointed observations and found to be in the range of 57.1-57.25 $\mathrm{mHz}$, showing a clear spin-up trend during the 2009 giant X-ray outburst.

Pulse profiles were generated by folding the light curves at respective spin period of the pulsar. This was done to understand the evolution of the pulse profile with luminosity and as the outburst progressed. While generating the pulse profiles for all the pointed observations, we chose the folding epoch close to the start time of the respective observation and then adjusted in such a way that the minima of the pulse profile align at phase zero. Some of the representative pulse profiles in 2-60 keV range are shown in Fig. 2. These profiles are arranged as the outburst progressed. The numbers quoted in each panel of the figure represent the beginning of the $R X T E$ observation (in MJD; left) and the estimated flux in $3-30 \mathrm{keV}$ range from spectral fitting (see Section 3.2 and Table 2 ; right). A careful investigation of the shape of the pulse profiles shown in Fig. 2 indicated a systematic evolution with the source flux. At lower intensity, the pulse profiles appeared as double peaked. Among these, the peak appearing in 0-0.5 phase range evolved and gradually splitted into two components as the outburst progressed towards the peak (MJD 55146-55167). This effectively produces a triple peaked profile at a flux level of $\geqslant 3.2 \times 10^{-9} \mathrm{erg} \mathrm{s}^{-1} \mathrm{~cm}^{-2}$. Assuming the source distance as $11 \mathrm{kpc}$ (Grindlay et al. 1984; İnam et al. 2004), corresponding 3-30 keV luminosity of the pulsar was estimated to be $4.6 \times 10^{37} \mathrm{erg} \mathrm{s}^{-1}$. The pulsar then restored back to its double peaked pulse profile shape as the outburst faded. We also noticed that the shape of profile was 
consistent at comparable luminosities irrespective of rising and declining phases of the outburst (see Fig. 2). Though a double peaked structure of the pulse profile has been already seen in 2S 1417-624 (Finger et al. 1996; Inam et al. 2004), the evolution of double-peaked profile to triple-peaked profile makes the present study interesting to explore it further through spectral studies.

We also investigated the evolution of pulse profile with energy at two different luminosities of the pulsar i.e. one during the onset of the outburst (MJD 55144.28) and the other at the peak of the outburst (MJD 55152.20). As described earlier, the source and background light curves in various energy ranges were extracted from the RXTE pointed observations on both the days, followed by barycenter correction on the background subtracted light curves and then folded with corresponding pulse period of the pulsar. Energy-resolved pulse profiles of the pulsar, obtained from both the observations are presented in Fig. $3 \&$ \& . We found that the pulse profiles of the pulsar are strongly energy dependent at different luminosities. The energy evolution of the first peak (0-0.5 phase range) in pulse profile was found to be faster than the second peak at late phases (0.5-1.0 phase range). Moreover, we also remarked that the energy evolution of pulse profile at lower luminosity was relatively simpler (Fig. 3) as compared to the brightest observation at the peak of the outburst (Fig. 4). In the latter observation, triple peaks are observed in the profile at energies below $15 \mathrm{keV}$ (Fig. 4). With increasing energy, the peak between 0.0-0.2 phase range gradually disappeared from the pulse profile in hard X-rays. We also noticed that the peak in 0.2-0.5 phase range was strongly energy dependent that evolved into a narrow component at higher energy, as seen in case of lower luminosity (Fig. 3). The prominent evolution of this component led to a minor phase shift of $\sim 0.1$ phase between the minima of soft $(2-6 \mathrm{keV})$ and hard X-ray profiles (above $30 \mathrm{keV}$ ). As in case of the profiles at lower luminosity, a broad structure in 0.5-1.0 phase range was also detected. This component became narrower with energy though the peak intensity was almost constant across the energy ranges. Pulsations were detected up to $\sim 60 \mathrm{keV}$ in both representative pulse profiles at different luminosities of the pulsar.

In order to quantify the fraction of X-ray photons contributing to the pulsation, we estimated pulse fraction in 2-60 keV range for all the observations and shown in Fig. 5 along with the source flux. We calculated the pulse fraction by considering a ratio between the difference of maximum and minimum intensity and the sum of maximum and minimum intensity in the pulse profiles. A negative correlation between the pulse fraction of the pulsar and the unabsorbed source flux in 3-30 keV range can be clearly seen in Fig. 5.

\subsection{Phase-averaged Spectroscopy}

We carried out pulse phase-averaged spectroscopy by using all available $R X T E$ observations of the pulsar during the 2009 giant outburst. The source and background spectra were extracted by following the procedures as described in Section 2. Spectral fitting was carried out by using XSPEC package of version 12.8.2. Data from PCA instrument (3-30 $\mathrm{keV}$ range) were used in our analysis. However, at the peak of the outburst, data from HEXTE instrument (15-70 keV range) were also used in our spectral fitting. As suggested by

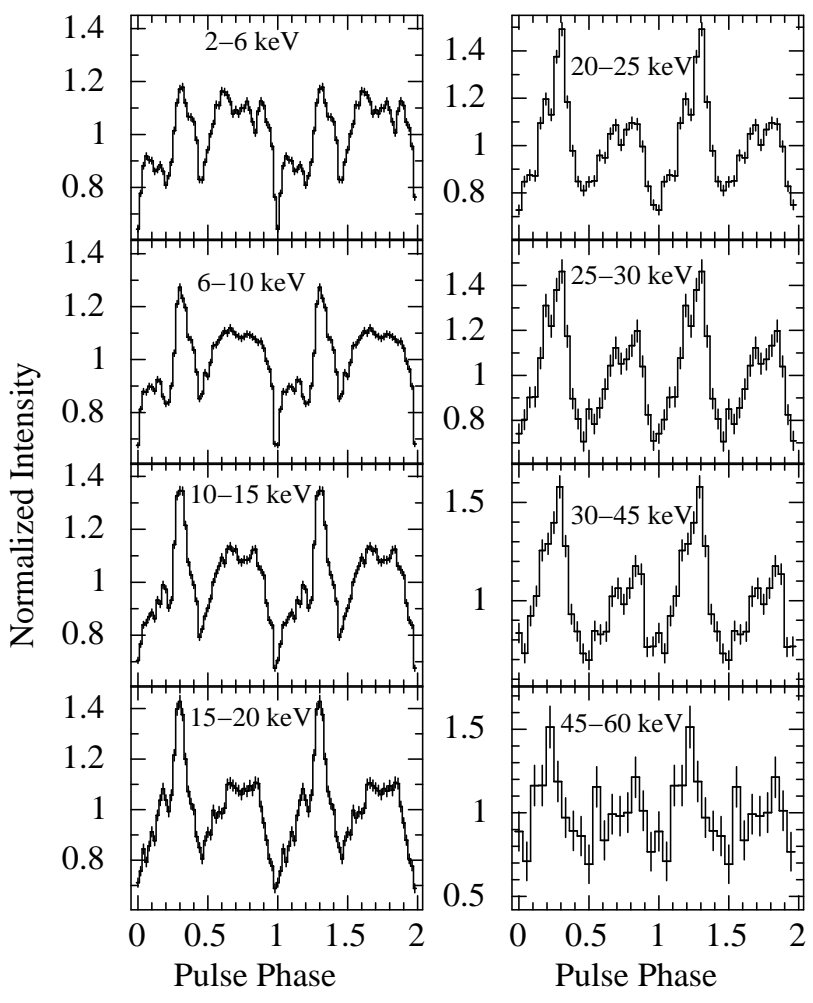

Figure 3. Energy resolved pulse profiles of 2S 1417-624 obtained from the RXTE observation on 2009 November 9 (MJD 55144.28) at rising phase of the Type-II outburst. A double-peaked profile can be clearly seen in all the panels of the figure. The error-bars represent $1 \sigma$ uncertainties. Two pulses in each panel are shown for clarity.

the instrument team, a systematic error of $1.0 \%$ was added to the pulsar spectra obtained from the RXTE/PCA. Various conventional models such as negative and positive exponential cutoff (NPEX), Fermi-Dirac cutoff (FDCut), cutoff power law and high-energy cutoff power law continuum models were attempted to fit the $3-30 \mathrm{keV}$ energy spectrum. Though a physical CompTT model was used to fit the spectrum, this model yielded a poor fit with a reduced- $\chi^{2} \geqslant 2$. A fluorescent iron emission line at $\sim 6.4 \mathrm{keV}$ was also detected in the pulsar spectrum. It was found that an absorbed cutoff power law and high-energy cutoff power law models along with the Gaussian function for iron emission line fitted the spectra obtained from all the $R X T E$ observations of the pulsar well. A representative broad-band spectrum in 3-70 keV range, obtained from the PCA and HEXTE data of the RXTE observation of the pulsar near the peak of the outburst (Obs. ID: 94032-02-03-03) is shown in Fig. 6. The spectral parameters obtained from fitting the $3-30 \mathrm{keV}$ PCA spectra from all the available $R X T E$ observations of the pulsar with the absorbed cutoff power law continuum model are presented in Table 2.

Change in spectral parameters of the pulsar with the 3-30 keV unabsorbed flux, obtained from fitting the $R X T E$ spectra with a cutoff power law continuum model are shown in Fig. 7. The parameters such as power-law photon index and cutoff energy are found to show interesting variations with the 3-30 keV unabsorbed flux. The pulsar spectrum became hard with the increase in luminosity. At the same time, the cutoff energy was also increased with the increase 

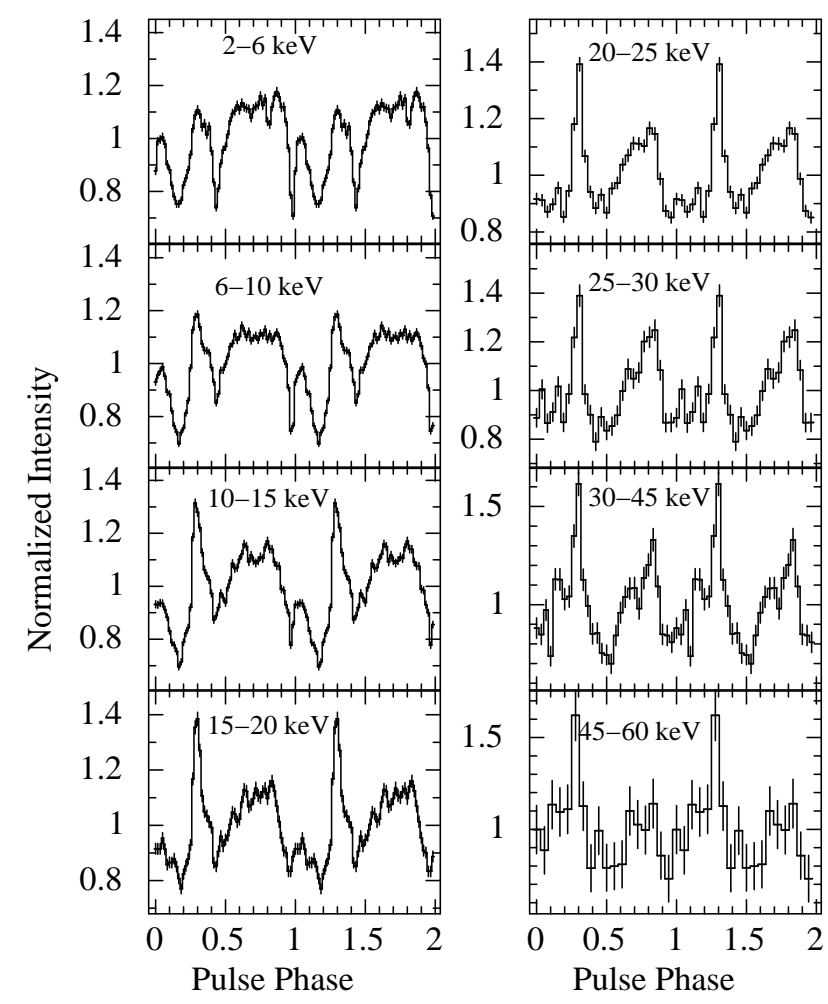

Figure 4. Energy resolved pulse profiles of $2 \mathrm{~S}$ 1417-624 obtained from the RXTE observation on 2009 November 17 (MJD $55152.20)$ near the peak of the Type-II outburst. A triple-peaked profile can be clearly visible in soft X-rays profiles that evolved into a double peaked structure at higher energies. The error-bars represent $1 \sigma$ uncertainties. Two pulses in each panel are shown for clarity.

in luminosity. Apart from the photon index and cutoff energy, the column density $\left(\mathrm{N}_{H}\right)$ which is variable in the range of $(1-6) \times 10^{22} \mathrm{~cm}^{-2}$ was found to be anti-correlated with the source flux. The estimated values of $\mathrm{N}_{H}$ was found to be marginally higher than the value of absorption column density in the direction of the source. The cutoff energy did not show any dependence on the power-law photon index.

\section{Phase-resolved Spectroscopy}

To understand the emission geometry, cause of pulse profile evolution from double-peaked to triple-peaked profile and investigate the changes in spectral parameters over pulse phases, we performed phase-resolved spectroscopy of the pulsar by using RXTE observations at rising phase and peak of the giant outburst. For this, the source and background spectra were extracted from GoodXenon mode data of PCA by following the standard procedure described in $R X T E$ cookbook and in RXTE Guest Observer Facility ${ }^{1}$. We selected two representative observations with Obs. IDs 9403202-01-06 (rising phase of the outburst) and 94032-02-03-04 (peak of the outburst) to extract phase-resolved spectra. Using appropriate background and response files, the $3-30 \mathrm{keV}$ spectrum for each phase bin was fitted with a high energy

${ }^{1}$ https://heasarc.gsfc.nasa.gov/docs/xte/recipes/pulse_phase.html

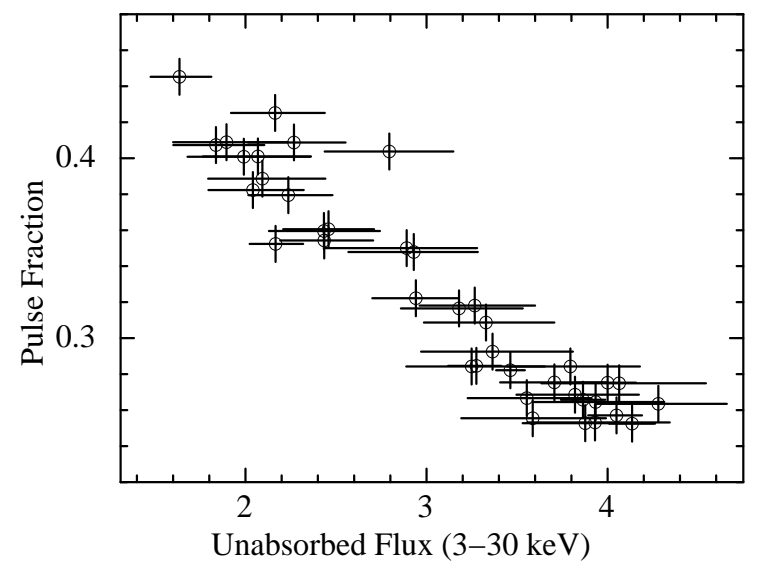

Figure 5. Pulse fraction (ratio between the difference of maximum and minimum intensity and the sum of maximum and minimum intensity in the pulse profile of the pulsar) with the 3-30 $\mathrm{keV}$ unabsorbed flux (in $10^{-9} \mathrm{erg} \mathrm{s}^{-1} \mathrm{~cm}^{-2}$ units) obtained from spectral fitting of the data from $R X T E / \mathrm{PCA}$ observations. The associated errors are calculated for $1 \sigma$ confidence level.

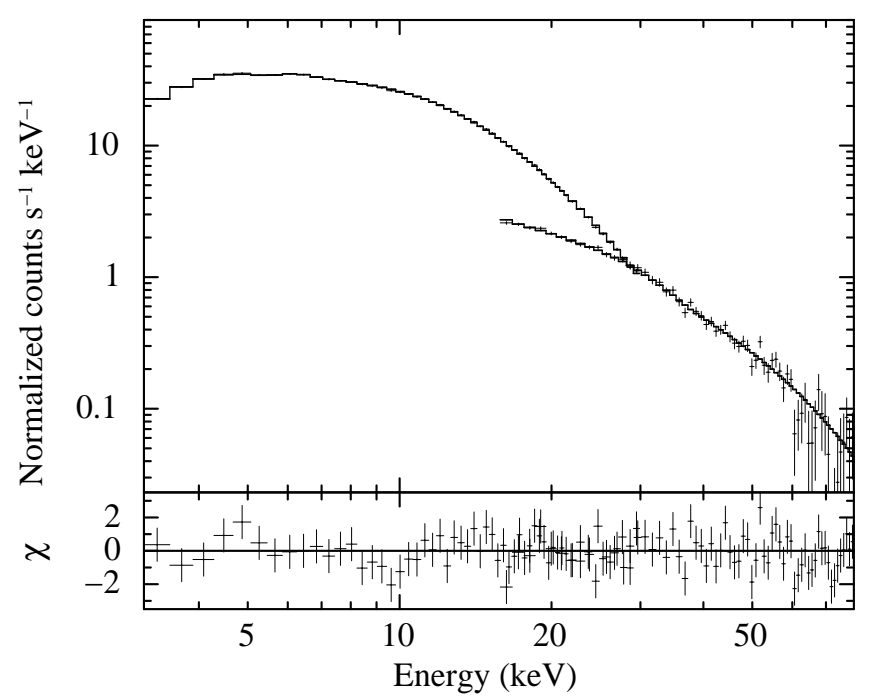

Figure 6. Broad-band spectra of $2 \mathrm{~S} 1417-624$ in the $3-70 \mathrm{keV}$ energy range (at the peak of the outburst; Obs-ID : 94032-0203-03) and the best-fit model consisting of a cutoff power law model along with a Gaussian function at $6.4 \mathrm{keV}$ for iron emission line are shown in the top panel. The bottom panel shows the contributions of the residuals to the $\chi^{2}$.

cutoff power law as well as cutoff power law continuum models along with a Gaussian function for iron emission line, as in case of phase-averaged spectroscopy. While fitting, the width of iron emission line was kept fixed at $0.1 \mathrm{keV}$. A systematic error of $1 \%$ was added to the phase-resolved spectra. We found that both the models yielded acceptable fits with comparable spectral parameters. Phase-resolved spectroscopy of RXTE observations of the pulsar at the rising phase and at the peak of the 2009 giant outburst, however, did not yield any significant and systematic variation of spectral parameters over the pulse phases. The unabsorbed flux in 3-30 keV range was found to follow similar pattern with pulse phase as that of the $3-10 \mathrm{keV}$ and $10-30 \mathrm{keV}$ pulse profiles of the pulsar. Other parameters such as power-law 
Table 2. Best-fitting spectral parameters of $2 \mathrm{~S}$ 1417-624 obtained from fitting the $R X T E / \mathrm{PCA}$ data during the 2009 giant outburst with a cutoff power-law model. The errors are quoted for $90 \%$ confidence level.

\begin{tabular}{|c|c|c|c|c|c|c|c|}
\hline Obs-id & $\mathrm{N}_{H}{ }^{a}$ & $\begin{array}{l}\text { Photon } \\
\text { index }\end{array}$ & $\begin{array}{c}\mathrm{E}_{\text {cut }} \\
(\mathrm{keV})\end{array}$ & $\begin{array}{c}\text { Line energy } \\
(\mathrm{keV})\end{array}$ & $\begin{array}{l}\text { Eq. width } \\
(\mathrm{eV})\end{array}$ & $\begin{array}{l}\text { Reduced- } \chi^{2} \\
\text { (d.o.f) }\end{array}$ & $\begin{array}{c}\text { Unabsorbed flux } \\
\quad(3-30 \mathrm{keV})\end{array}$ \\
\hline 94032-02-01-00 & $3.2 \pm 0.9$ & $0.41 \pm 0.09$ & $14.6 \pm 1.4$ & $6.52 \pm 0.27$ & $55 \pm 28$ & $1.17(43)$ & $1.89 \pm 0.34$ \\
\hline 94032-02-01-02 & $3.2 \pm 0.6$ & $0.39 \pm 0.06$ & $15.4 \pm 0.9$ & $6.34 \pm 0.21$ & $128 \pm 60$ & $1.10(42)$ & $2.79 \pm 0.35$ \\
\hline 94032-02-01-06 & $2.1 \pm 0.5$ & $0.23 \pm 0.05$ & $13.7 \pm 0.7$ & $6.43 \pm 0.15$ & $71 \pm 22$ & $0.50(47)$ & $2.43 \pm 0.27$ \\
\hline 94032-02-02-00 & $1.4 \pm 0.6$ & $0.17 \pm 0.06$ & $14.1 \pm 0.8$ & $6.26 \pm 0.16$ & $133 \pm 78$ & $1.01(45)$ & $2.89 \pm 0.38$ \\
\hline 94032-02-02-01 & $2.1 \pm 0.6$ & $0.34 \pm 0.06$ & $17.4 \pm 1.2$ & $6.35 \pm 0.18$ & $80 \pm 50$ & $0.84(48)$ & $2.92 \pm 0.35$ \\
\hline 94032-02-02-02 & $1.9 \pm 0.6$ & $0.24 \pm 0.05$ & $16.1 \pm 0.9$ & $6.28 \pm 0.19$ & $60 \pm 23$ & $0.89(50)$ & $3.26 \pm 0.33$ \\
\hline $94032-02-02-03$ & $1.4 \pm 0.7$ & $0.20 \pm 0.06$ & $15.6 \pm 1.2$ & $6.32 \pm 0.12$ & $90 \pm 23$ & $0.67(49)$ & $3.24 \pm 0.41$ \\
\hline 94032-02-03-00 & $1.5 \pm 0.5$ & $0.20 \pm 0.05$ & $15.9 \pm 0.9$ & $6.26 \pm 0.17$ & $71 \pm 22$ & $1.04(50)$ & $3.55 \pm 0.35$ \\
\hline 94032-02-03-01 & $1.7 \pm 0.5$ & $0.27 \pm 0.05$ & $17.4 \pm 1.0$ & $6.38 \pm 0.15$ & $75 \pm 22$ & $1.15(50)$ & $3.87 \pm 0.37$ \\
\hline $94032-02-03-03$ & $1.1 \pm 0.2$ & $0.20 \pm 0.02$ & $16.3 \pm 0.5$ & $6.18 \pm 0.21$ & $120 \pm 60$ & $0.72(50)$ & $3.99 \pm 0.15$ \\
\hline 94032-02-03-04 & $1.1 \pm 0.2$ & $0.17 \pm 0.02$ & $15.6 \pm 0.5$ & $6.18 \pm 0.19$ & $103 \pm 50$ & $0.65(49)$ & $4.04 \pm 0.14$ \\
\hline 94032-02-03-05 & $1.8 \pm 0.7$ & $0.27 \pm 0.06$ & $17.4 \pm 1.2$ & $6.39 \pm 0.17$ & $61 \pm 24$ & $0.85(49)$ & $4.06 \pm 0.47$ \\
\hline 94032-02-04-01 & $1.4 \pm 0.3$ & $0.28 \pm 0.01$ & $18.4 \pm 0.6$ & $6.30 \pm 0.16$ & $98 \pm 37$ & $0.67(49)$ & $4.13 \pm 0.12$ \\
\hline 94032-02-04-02 & $1.0 \pm 0.5$ & $0.25 \pm 0.05$ & $16.9 \pm 1.1$ & $6.28 \pm 0.15$ & $75 \pm 23$ & $0.71(46)$ & $3.93 \pm 0.41$ \\
\hline 94032-02-05-00 & $1.4 \pm 0.4$ & $0.28 \pm 0.04$ & $16.7 \pm 0.7$ & $6.31 \pm 0.13$ & $69 \pm 31$ & $0.86(49)$ & $3.70 \pm 0.29$ \\
\hline 94032-02-05-01 & $1.3 \pm 0.5$ & $0.30 \pm 0.05$ & $17.8 \pm 1.0$ & $6.39 \pm 0.16$ & $67 \pm 22$ & $0.81(50)$ & $3.93 \pm 0.37$ \\
\hline 94032-02-05-02 & $1.1 \pm 0.2$ & $0.22 \pm 0.02$ & $15.9 \pm 0.5$ & $6.29 \pm 0.21$ & $71 \pm 32$ & $0.57(49)$ & $3.86 \pm 0.12$ \\
\hline $94032-02-05-03$ & $1.1 \pm 0.5$ & $0.27 \pm 0.05$ & $16.3 \pm 0.9$ & $6.22 \pm 0.21$ & $66 \pm 23$ & $0.63(50)$ & $3.79 \pm 0.38$ \\
\hline 94032-02-05-04 & $1.2 \pm 0.5$ & $0.26 \pm 0.04$ & $16.4 \pm 0.9$ & $6.31 \pm 0.11$ & $81 \pm 18$ & $0.79(49)$ & $3.82 \pm 0.35$ \\
\hline 94032-02-06-00 & $1.5 \pm 0.6$ & $0.32 \pm 0.05$ & $17.2 \pm 1.1$ & $6.37 \pm 0.16$ & $82 \pm 42$ & $0.83(48)$ & $3.58 \pm 0.4$ \\
\hline 94032-02-06-01 & $1.0 \pm 0.4$ & $0.40 \pm 0.02$ & $17.5 \pm 0.7$ & $6.39 \pm 0.16$ & $64 \pm 39$ & $0.55(47)$ & $3.27 \pm 0.13$ \\
\hline 94032-02-06-02 & $1.3 \pm 0.2$ & $0.22 \pm 0.01$ & $15.0 \pm 0.4$ & $6.45 \pm 0.12$ & $69 \pm 13$ & $0.88(48)$ & $3.46 \pm 0.08$ \\
\hline 94032-02-06-03 & $1.9 \pm 0.5$ & $0.32 \pm 0.06$ & $16.1 \pm 1.1$ & $6.50 \pm 0.25$ & $55 \pm 24$ & $0.92(50)$ & $3.32 \pm 0.37$ \\
\hline 94032-02-06-04 & $1.6 \pm 0.7$ & $0.24 \pm 0.07$ & $14.8 \pm 1.0$ & $6.24 \pm 0.14$ & $76 \pm 25$ & $0.79(49)$ & $3.36 \pm 0.44$ \\
\hline 94444-01-01-00 & $2.0 \pm 0.4$ & $0.33 \pm 0.04$ & $15.5 \pm 0.7$ & $6.53 \pm 0.14$ & $68 \pm 30$ & $1.05(45)$ & $2.94 \pm 0.23$ \\
\hline 94444-01-01-01 & $1.6 \pm 0.5$ & $0.24 \pm 0.05$ & $13.1 \pm 0.7$ & $6.44 \pm 0.16$ & $99 \pm 40$ & $0.91(48)$ & $2.45 \pm 0.25$ \\
\hline 94444-01-02-00 & $2.3 \pm 0.6$ & $0.29 \pm 0.06$ & $15.0 \pm 1.0$ & $6.39 \pm 0.16$ & $59 \pm 21$ & $0.53(47)$ & $3.17 \pm 0.35$ \\
\hline 94444-01-03-00 & $2.5 \pm 0.6$ & $0.38 \pm 0.06$ & $15.0 \pm 1.1$ & $6.57 \pm 0.23$ & $72 \pm 52$ & $1.11(48)$ & $2.43 \pm 0.3$ \\
\hline 94444-01-03-03 & $2.0 \pm 0.7$ & $0.30 \pm 0.07$ & $13.1 \pm 0.9$ & $6.47 \pm 0.27$ & $101 \pm 64$ & $0.99(43)$ & $2.06 \pm 0.29$ \\
\hline 94444-01-04-00 & $2.8 \pm 0.7$ & $0.41 \pm 0.07$ & $13.9 \pm 1.2$ & $6.59 \pm 0.32$ & $60 \pm 28$ & $1.27(45)$ & $1.83 \pm 0.26$ \\
\hline 94032-02-01-01 & $4.3 \pm 0.8$ & $0.5 \pm 0.07$ & $16.1 \pm 1.4$ & - & - & $1.12(47)$ & $2.04 \pm 0.28$ \\
\hline 94032-02-01-03 & $2.0 \pm 0.4$ & $0.17 \pm 0.05$ & $12.3 \pm 0.9$ & - & - & $1.02(45)$ & $2.16 \pm 0.15$ \\
\hline 94032-02-01-04 & $3.5 \pm 1.0$ & $0.35 \pm 0.1$ & $13.3 \pm 1.6$ & - & - & $0.73(51)$ & $1.99 \pm 0.36$ \\
\hline 94032-02-01-05 & $3.0 \pm 0.8$ & $0.33 \pm 0.09$ & $13.6 \pm 1.5$ & - & - & $1.04(47)$ & $2.09 \pm 0.34$ \\
\hline 94444-01-03-01 & $5.1 \pm 0.2$ & $0.56 \pm 0.06$ & $16.5 \pm 1.2$ & - & - & $0.87(50)$ & $2.26 \pm 0.28$ \\
\hline 94444-01-03-02 & $5.4 \pm 0.9$ & $0.56 \pm 0.06$ & $16.1 \pm 1.1$ & - & - & $0.85(50)$ & $2.16 \pm 0.27$ \\
\hline 94444-01-03-05 & $4.0 \pm 0.7$ & $0.48 \pm 0.06$ & $15.3 \pm 1.0$ & - & - & $1.27(47)$ & $2.23 \pm 0.24$ \\
\hline 94444-01-04-01 & $4.5 \pm 0.7$ & $0.53 \pm 0.06$ & $14.1 \pm 0.9$ & - & - & $0.86(47)$ & $1.63 \pm 0.17$ \\
\hline
\end{tabular}

${ }^{a}$ : Equivalent hydrogen column density in the unit of $10^{22} \mathrm{~cm}^{-2} ;{ }^{b}:$ in unit of $10^{-9} \mathrm{erg} \mathrm{s}^{-1} \mathrm{~cm}^{-2}$.

photon index, cutoff energy and absorption column density did not show any systematic variation with pulse phase for both the observations. Therefore, it is difficult to draw any meaningful conclusion from phase-resolved spectroscopy of the RXTE observations of the pulsar during the 2009 giant outburst.

\section{DISCUSSION AND CONCLUSIONS}

We have presented a comprehensive spectral and timing analysis of the pulsar $2 \mathrm{~S} 1417-624$ by using $R X T E$ observations during a giant outburst in 2009. The most intriguing aspect of our study is that, we witnessed peculiar changes in the pulse profiles of the pulsar for the first time. A double peaked profile at lower luminosity was found to evolve into a triple peaked structure with the increase in luminosity. This kind of variation has not been seen in the pulsar during any other Type I and Type II outbursts (Finger et al. 1996; Inam et al. 2004). During most of the earlier observations, pulse profiles were double-peaked and showed a marginal en- ergy dependence. A simple broad profile was also reported at lower luminosity during the 1999 outburst (Raichur \& Paul 2010). This establishes the fact that the pulsar 2 S 1417-624 showed luminosity dependent pulse profiles from a single peak to multiple peaks across a wide range of flux starting from $10^{-11}$ to $10^{-9} \mathrm{erg} \mathrm{s}^{-1} \mathrm{~cm}^{-2}$ (İnam et al. 2004; Raichur \& Paul 2010; and the present work).

It has been already noticed that Be/X-ray binary pulsars such as EXO 2030+375 (Naik et al. 2013; Naik \& Jaisawal 2015; Epili et al. 2017), GX 304-1 (Jaisawal et al. 2016) show multiple peaks in the pulse profile during outbursts. These peaks are known to be strongly energy dependent and usually associated with manifold minima or dip like structures in the soft X-rays. Phase-resolved spectroscopy of observations during X-ray outbursts of these transient pulsars showed the presence of additional matter at dip phases. This indicated that the dips in the pulse profiles are originated due to the absorption/obscuration of X-ray photons by narrow streams of matter that are phase-locked with the pulsar. As a result, the multiple peaks/dips observed in the pulse profiles of 


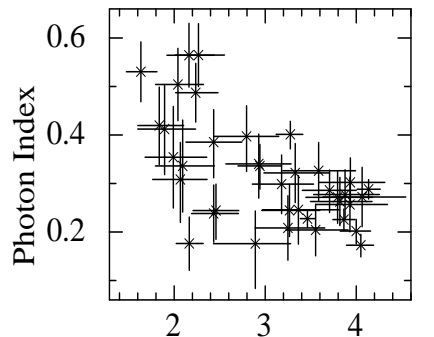

Flux (3-30 keV)

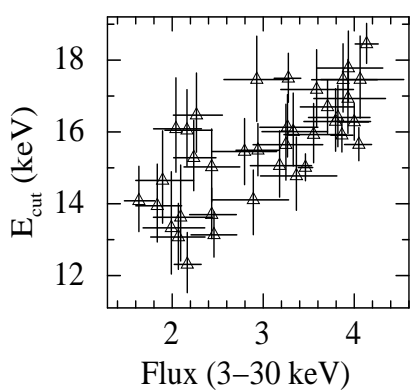

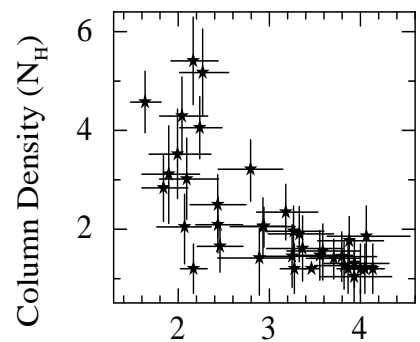

Flux (3-30 keV)

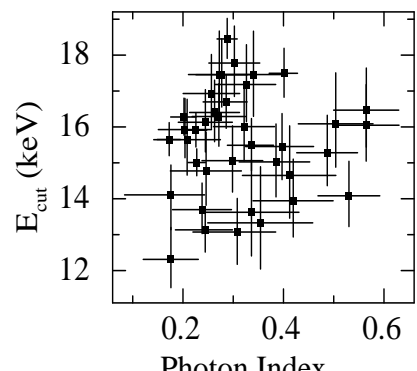

Figure 7. Variation of spectral parameters such as power-law photon index, absorption column density, cutoff energy with unabsorbed source flux in 3-30 keV range, obtained from the fitting of $R X T E / \mathrm{PCA}$ data with a cutoff power law continuum model. The error-bars are estimated for $90 \%$ confidence level.

transient X-ray pulsars e.g. EXO 2030+375 and GX 304-1 are associated with the effect of partial absorption of the radiation beam rather than originating from different hot spots on the neutron star surface.

In the present study, we have observed the evolution of a broad peak (in 0-0.5 phase range; Fig. 2) in the pulse profile into two peaks at a flux level of $\sim 3 \times 10^{-9} \mathrm{erg} \mathrm{s}^{-1} \mathrm{~cm}^{-2}$. This effectively evolved into three peaks at brighter phases of the giant outburst. Nevertheless, the source intensity was found to be relatively high i.e. $\sim 2.7 \times 10^{-9} \mathrm{erg} \mathrm{s}^{-1} \mathrm{~cm}^{-2}$ in $3-20 \mathrm{keV}$ range during the 2009 outburst compared to the previous events when the peak flux of the pulsar was observed to be $\sim 1.7 \times 10^{-9} \mathrm{erg} \mathrm{s}^{-1} \mathrm{~cm}^{-2}$ in above energy range (İnam et al. 2004). Therefore, a double peaked profile was only observed in the earlier studies. To explore the origin of multiple peaks at brighter phases of the pulsar, we have performed phase-resolved spectroscopy at two different luminosities of the pulsar. Our results showed moderate variation in the column density across the pulse phases in contrast to other Be/X-ray binary sources. Though, the observed column density $\left(1-5 \times 10^{22} \mathrm{~cm}^{-2}\right)$ was found to be marginally higher than the Galactic value $\left(\sim 1.4 \times 10^{22} \mathrm{~cm}^{-2}\right)$ along the line of sight, it is not sufficient to produce a remarkable dip or affect the beam function up to $\sim 20 \mathrm{keV}$, as seen in Fig. 4. Limited low energy coverage of the RXTE/PCA (3 $\mathrm{keV}$ ) may be one of the reason that constrained the column density measurement. Future studies with sensitive soft Xray instruments such as NICER (Gendreau et al. 2012) can explore the surrounding of the pulsar in depth.

We have noticed distinct luminosity dependence of the pulse profiles during the 2009 outburst. By assuming a source distance of $11 \mathrm{kpc}$ (Grindlay et al. 1984; İnam et al. 2004), the 3-30 keV unabsorbed luminosity can be calculated to be in the range of $\sim(2.4-6) \times 10^{37} \mathrm{erg} \mathrm{s}^{-1}$ during the 2009
Type-II outburst. Moreover, for a distance of $5 \mathrm{kpc}$, pulsar luminosity falls to a range of $4.8 \times 10^{36}$ to $1.2 \times 10^{37} \mathrm{erg} \mathrm{s}^{-1}$. For above distances, the source luminosity was estimated to be in the order of $10^{37} \mathrm{erg} \mathrm{s}^{-1}$ which is a typical value of critical luminosity of accretion powered X-ray pulsars (Becker et al. 2012; Reig \& Nespoli 2013; Mushtukov et al. 2015). Critical luminosity of an accretion powered X-ray pulsar is crucial to understand the transition between two accretion regimes i.e. sub-critical and super-critical phases (Basko \& Sunyaev 1976; Becker et al. 2012). At lower luminosity (sub-critical phase), accretion flow is expected to halt by Coulomb interaction close to the neutron star surface. In this regime, the high energy emission is dominated by a pencil beam (propagating parallel to magnetic field) or pulsed component (Basko \& Sunyaev 1976). This component may produce a single or double peaked pulse profile depending on the visibility of the emission regions at poles of the neutron star. With increasing mass accretion rate, the radiation pressure takes lead and up-scatters the accreting plasma particles in a presence of radiation dominated shock above the surface. X-ray photons emitted in this situation mostly diffuse through the side wall of the accretion column in the form of a fan beam pattern (perpendicular to magnetic field lines). This kind of pattern is un-pulsed and may produce a complex pulse profile. Becker et al. (2012) has calculated the critical luminosity by considering various physical processes in the accretion column which can be expressed as

$$
\begin{aligned}
L_{\text {crit }}= & 1.49 \times 10^{37} \mathrm{erg} \mathrm{s}^{-1}\left(\frac{\Lambda}{0.1}\right)^{-7 / 5} w^{-28 / 15} \\
& \times\left(\frac{M}{1.4 \mathrm{M}_{\odot}}\right)^{29 / 30}\left(\frac{R}{10 \mathrm{~km}}\right)^{1 / 10}\left(\frac{B}{10^{12} \mathrm{G}}\right)^{16 / 15}(1)
\end{aligned}
$$

where $R, M$ and $B$ are the radius, mass and magnetic field (in $10^{12} \mathrm{G}$ ) of the neutron star, respectively. The constant $\Lambda$ characterizes the mode of accretion which is assumed as 0.1 (for disk accretion) in our case. While the parameter $\omega$ represents the shape of the photon spectrum and considered as 1 (Becker \& Wolff 2007). For standard case, above equation reduces to

$$
L_{\text {crit }}=1.49 \times 10^{37}\left(\frac{B}{10^{12} \mathrm{G}}\right)^{16 / 15} \mathrm{erg} \mathrm{s}^{-1}
$$

Therefore, the critical luminosity for the pulsar 2 S 1417 624 can be calculated to be $\sim 1.33 \times 10^{37}$ erg $s^{-1}$ by assuming a magnetic field strength of $9 \times 10^{11} \mathrm{G}$ as reported by Inam et al. (2004). The estimated value of critical luminosity matches well with the pulsar luminosity observed during the 2009 giant outburst for considered distances. It also indicates that the pulsar was emitting at the critical luminosity or above the critical luminosity near the peak of the outburst. For sub-critical (below critical luminosity) sources, emission geometry is expected to be simple i.e. described by a pencil beam pattern from pulsating component. Thus, the presence of double peaks with individual peaks separated by 0.5 pulse phase in the profile suggests a simple beam function originated from both the poles of the neutron star during outburst. As the source approaches the critical luminosity, the contribution from un-pulsed photons escaping through side walls of the column increases. This increase may influence the total beam geometry and produce multiple peaks in 
the pulse profile, as seen in our study. This interpretation is further supported by the observed anti-correlation between pulsed fraction and source flux, as shown in Fig. 5. A higher pulse fraction is expected from a pulsar in case of directly beamed emission. While the increasing contribution of the fan beam enhances the un-pulsed component or decreases the pulsed component at higher luminosity from the pulsar. This results a negative correlation in the pulse fraction with luminosity, as observed in our study. Other sources in luminosity range of $10^{35}-10^{37} \mathrm{erg} \mathrm{s}^{-1}$ e.g. SXP 1323 (Yang et al. 2018) have also shown a negative trend with source intensity. Furthermore, the reflection from neutron star surface can also contribute to un-pulsed radiations at higher luminosity (Mushtukov et al. 2018). Therefore, we suggest that the changes in pulse morphology from a double to a triple peaked profile is associated with the change in beam pattern near the critical luminosity.

In the support of results obtained from timing studies, we explored the spectral properties of the pulsar by using RXTE data during the giant outburst. The $3-30 \mathrm{keV}$ spectrum of $2 \mathrm{~S}$ 1417-624 was found to be well described by a cutoff power law model along with a Gaussian function at $6.4 \mathrm{keV}$ for the iron emission line. We studied changes in the spectral shape with the pulsar luminosity during the 2009 giant outburst. The photon index was found to be anticorrelated with the unabsorbed flux (see Fig. 7), indicating that the spectrum became harder during the bright phase of the outburst. Interestingly, the observed power-law photon index during the 2009 outburst (in 0.1-0.6 range) is found to be lower than the earlier reported values (in 0.8-2.5 range) by İnam et al. (2004). The authors also found a clear negative correlation in photon index with flux in (0.01-1) $\times 10^{-9}$ $\mathrm{erg} \mathrm{s}^{-1} \mathrm{~cm}^{-2}$ range as in the present case. Along with negative correlation between the photon index and source flux, the values of photon index are found to be clustered in (3-4) $\times 10^{-9} \mathrm{erg} \mathrm{s}^{-1} \mathrm{~cm}^{-2}$ flux range.

The pattern of distribution of power-law photon index with source flux (Fig. 7) has been observed in pulsars accreting in sub-critical regime or close to the critical luminosity (Reig \& Nespoli 2013; Postnov et al. 2015; Epili et al. 2017 and references therein). The height of the pulsar emission region is considered to be in the range of a few kilometers (Becker et al. 2012). With increase in mass accretion rate, the height of the emission region gets reduced. At the same time, increase in the optical depth of the accretion column leads to the origin of harder spectra (or negative correlation) in the sub-critical regime. Nevertheless, the cutoff energy also showed a positive trend with the source flux. This parameter is associated with the plasma temperature and indicates the temperature enhancement of emission region with increasing luminosity (Soong et al. 1990; Unger et al. 1992). The presence of clustered values of photon index in (3-4) $\times$ $10^{-9} \mathrm{erg} \mathrm{s}^{-1} \mathrm{~cm}^{-2}$ flux range can be associated with the transition from sub-critical to super-critical regimes during the giant outburst. Therefore, the results from our spectral analysis also support the idea of accretion transition close to the critical luminosity of the pulsar 2S 1417-624 during the giant outburst. Accretion powered X-ray pulsars have strong magnetic field in the order of $\sim 10^{12} \mathrm{G}$. Detection of absorption features in 10-100 keV range pulsar spectrum, known as cyclotron resonance scattering features, provides direct estimation of the pulsar magnetic field (Jaisawal \& Naik 2017).
However, in the present case, no such features were detected in the pulsar spectra even at the peak of the giant outburst. Future observations with instruments covering broad energy range may provide an opportunity to constrain the magnetic field of the pulsar.

In summary, we have studied spectral and timing properties of 2S 1417-624 during a Type II outburst in 2009. The pulse profile was found to evolve from a double-peaked profile to triple-peaked profile with the increase in the source luminosity. Spectral studies of the RXTE observations during the giant outburst showed that the pulsar was accreting close to the critical luminosity level. This led to the changes in the beam pattern, effectively from a pencil beam to a mixture of pencil and fan beams. This is also supported by variation in the pulse fraction with luminosity as well as results obtained from phase-resolved spectroscopy. We did not find any specific pattern in the column density with pulse phase of the pulsar indicating that the change in pattern of the pulse profile is not associated with the inhomogeneous distribution of matter (in the form of narrow accretion streams) around the poles. We suggest that the three-peaked profile at brighter phases of outburst is caused due to the change in beam pattern at the critical luminosity in contrast to other effects such as absorption by a narrow stream of matter or emission from multiple hot spots on the surface.

\section{ACKNOWLEDGMENTS}

We thank the referee for his/her suggestions on the paper. The research work at Physical Research Laboratory is funded by the Department of Space, Government of India. GKJ acknowledges support from the Marie Skłodowska-Curie Actions grant no. 713683 (H2020; COFUNDPostdocDTU). This research has made use of data obtained through HEASARC Online Service, provided by the NASA/GSFC, in support of NASA High Energy Astrophysics Programs.

\section{REFERENCES}

Apparao K. M. V., Naranan S., Kelley R. L., Bradt H. V., 1980, A\&A, 89, 249

Basko M. M., Sunyaev R. R., 1976, MNRAS, 175, 395

Becker P. A. et al., 2012, A\&A, 544, A123

Becker P. A., Wolff M. T., 2007, ApJ, 654, 435

Epili P., Naik S., Jaisawal G. K., Gupta S., 2017, MNRAS, 472, 3455

Finger M. H., Wilson R. B., Chakrabarty D., 1996, A\&AS, 120, 209

Gendreau K. C., Arzoumanian Z., Okajima T., 2012, SPIE, 8443, 13

Giacconi R., Gursky H., Kellogg E., Schreier E., Tananbaum H., 1971, 167, 67

Grindlay J. E., Petro L. D., McClintock J. E., 1984, ApJ, 276, 621

Hewish A., Bell S. J., Pilkington J. D. H., Scott P. F., Collins R. A., 1968, Nature, 217, 709

İnam S. Ç., Baykal A., Matthew Scott D., Finger M., Swank J., 2004, MNRAS, 349, 173

Jahoda K., Swank J. H., Giles A. B., Stark M. J., Strohmayer T., Zhang W., Morgan E. H., 1996, in Proc. SPIE, Vol. 2808, 
EUV, X-Ray, and Gamma-Ray Instrumentation for Astronomy VII, Siegmund O. H., Gummin M. A., eds., pp. 59-70 Jaisawal G. K., Naik S., Epili P., 2016, MNRAS, 457, 2749

Jaisawal G. K., Naik S., 2017, 7 years of MAXI: monitoring X-ray Transients Conference proceeding, 153 (arXiv:1705.05536)

Kelley R. L., Doxsey R. E., Jernigan J. G., Rappaport S., Apparao K. M. V., Naranan S., 1981, ApJ, 243, 251

Krimm H. A. et al. 2009, ATel, 2276

Krimm et al., 2013, ApJSS 209,14

Krimm H. A. et al. 2018, ATel, 11569

Leahy D.A., A\&A, 1987, 180, 275

Levine A. M., Bradt H., Cui W., Jernigan J. G., Morgan E. H., Remillard R., Shirey R. E., Smith D. A., 1996, ApJ, 469, L33

Mushtukov A. A., Suleimanov V. F., Tsygankov S. S., Poutanen J., 2015, MNRAS, 447, 1847

Mushtukov A. A., Verhagen P. A., Tsygankov S. S., van der Klis M., Lutovinov A. A., Larchenkova T. I., 2018, MNRAS, 474, 5425

Naik S. et al., 2008, ApJ, 672, 516

Naik S., Paul B., Kachhara C., Vadawale S. V., 2011, MNRAS, 413, 241

Naik S., Maitra C., Jaisawal G. K., Paul B., 2013, ApJ, 764, 158

Naik S., Jaisawal G. K., 2015, Research in Astronomy and Astrophysics, 15,537

Nakajima M. et al. 2018, ATel, 11479

Negueruela I., Reig P., Coe M. J., Fabregat J., 1998, A\&A, 336, 251

Paul B., Naik S., 2011, Bulletin of the Astronomical Society of India, 39, 429

Porter J. M., Rivinius T., 2003, PASP, 115, 1153

Postnov K. A., Gornostaev M. I., Klochkov D., Laplace E., Lukin V. V., Shakura N. I., 2015, MNRAS, 452, 1601

Reig P., 2011, Ap\&SS, 332, 1

Reig P., Nespoli E., 2013, A\&A, 551, A1

Raichur H., Paul B., MNRAS, 2010, 406, 2663

Rothschild R. E. et al., 1998, ApJ, 496, 538

Soong Y., Gruber D. E., Peterson L. E., Rothschild R. E., 1990, ApJ, 348, 641

Stella L., White N. E., Rosner R., 1986, ApJ, 308, 669

Tsygankov S. S., Wijnands R., Lutovinov A. A., Degenaar N., Poutanen J., 2017, MNRAS, 470, 126

Unger S. J., Norton A. J., Coe M. J., Lehto H. J., 1992, MNRAS, 256, 725

Yang J., Zezas A., Coe M. J., Drake J. J., Hong J., Laycock S. G. T., Wik D. R., 2018, MNRAS, in press 